\title{
Coaching aplicado a la hostelería y el turismo
}

\author{
Christian Gistain Montolío \\ Psicólogo. Coach. Profesor del Máster en Coaching y Liderazgo Universidad de Valencia \\ c.gistain@hotmail.com
}

\begin{abstract}
El sector de la hostelería y el turismo son vitales para España. Su historia y los datos actuales así lo reflejan. Perteneciendo así al denominado sector servicios, su éxito radica en el componente social de todos aquellos trabajadores y consumidores en lo que se refiere a las relaciones entre ambos y la forma de interacción que muestran.
\end{abstract}

Para ello, el coaching aplicado a este sector tendrá como objetivo optimizar los recursos de los empleados con el fin de lograr la satisfacción de los mismos, el desarrollo y la adquisición de nuevas habilidades, y la creación de equipos de trabajadores motivados y comprometidos con su tarea.

Por último, se desarrollará la idea que afirma que la correcta utilización de las redes sociales y las diferentes aplicaciones que internet ofrece, ha cobrado especial relevancia en la actualidad para poder aplicar tanto funciones propias del coaching como el mantenimiento de un contacto que afiance todo lo trabajado.

The hospitality industry and tourism are vital to Spain. Its history and current data reflect this. Thus belonging to the service sector, its success is based in the social component of all those workers and consumers in regard to relations between both and the way of interaction that show.

For this, the coaching applied to this sector will aim to optimize the resources of employees in order to achieve satisfaction thereof, development and acquisition of new skills, team building motivated workers and committed workers to their task.

Finally, the idea that states that the proper use of social networks and the internet offers various applications, has gained special relevance today to apply both functions of coaching as maintaining a contact fasten everything will run worked.

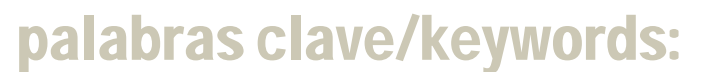

Hostelería, turismo, restauración, motivación, objetivos.

Hospitality, tourism, catering, motivation, objectives.

\section{1.- INTRODUCCIÓN.}

España es uno de los destinos turísticos preferidos tanto por turistas extranjeros como nacionales. La diversidad de sus gentes, sus culturas, sus regiones, su gastronomía, sus costumbres, su patrimonio histórico, arquitectónico y cultural hacen que cada año, millones de personas elijan nuestro país como destino para sus días de relax y di- versión. El buen clima y el sello de calidad por pertenecer a la Unión Europea, son el remate perfecto de la jugada.

Es a partir del final de la segunda guerra mundial, desde 1950 en adelante, cuando el turismo ha sido y es una de las mayores fuentes de ingresos para España, permitiendo así el desarrollo de numerosas empresas relacionadas con este sector. 
En la actualidad, numerosas entidades dedicadas al mundo de la hostelería lo saben. Aquellas que se inician o que desean mejorar su servicio, optan por contratar una figura de reciente aparición, el psicólogo o el responsable de realizar coaching de empresas dedicadas a este sector.

¿Cómo se puede aplicar la psicología y el coaching al mundo de la hostelería y el turismo? Como en todo negocio, empresa o servicio en el que haya personas o se requiera tratar con ellas, nos será de gran ayuda conocer el origen, el significado y las diversas consecuencias de lo que denominamos "la conducta humana". Por fortuna o por desgracia, vivimos en un mundo en el que el producto ofertado suele ser el mismo en la mayoría de los casos. Una bebida y sus combinados siempre proceden de una lata o botella de una determinada marca y saben igual en casi todas las partes del mundo. La forma de servirlas y de cómo se realiza esta acción, será la que diferencie un negocio del resto. Para ello, se ha de contar con un equipo de trabajadores bien formado, unido, motivado en su tarea y lo más importante en el sector de la hostelería, feliz. Las matemáticas no engañan. Un grupo de personas que se siente a gusto y que está alegre, tiene más probabilidades de contagiar esta emoción que uno que no lo está.

A continuación, se detallará la relevancia del turismo y de la hostelería en España, las formas de proceder de un responsable de coaching en el mundo de la hostelería, la necesidad de la formación como vía inicial de primera toma de contacto con el cliente, las claves para motivar a los trabajadores, el buen uso de las redes sociales para los negocios y el propio coaching,

\section{2.- DESARROLLO}

\section{1- RELEVANCIA DEL TURISMO Y DE LA HOSTELERÍA EN ESPAÑA.}

El turismo es toda aquella actividad económica que permite y explota los viajes que realiza una persona desde su punto actual de residencia a otro distinto. Un turista es aquella persona que se traslada de su domicilio habitual a otro punto geográfico, estando ausente de su lugar de residencia habitual más de 24 horas y realizando pernoctación en el otro punto geográfico. De no realizar dicha pernoctación, se le considera un excursionista.

Turismo exterior es aquel que realizan los residentes de un país a otro distinto, mientras que turismo interior es el que se realiza dentro de un mismo país, desplazándose por tanto a una región o localidad distinta a la de la residencia habitual. El que realiza la primera acción suele ser llamado turista extranjero, mientras que el que lleva a cabo la segunda, es conocido como turista nacional.

Existen por tanto, numerosas empresas dedicadas tanto al sector de la hostelería más directo como aquellas que están relacionadas con todo lo que implique dar servicio a las mismas.

Según datos del I.E.T. (Instituto de Estudios Turísticos) en su estudio de Balance del Turismo en España de 2010, el turismo supuso unos ingresos de más de 80.000 millones de euros, aportando así el 10.7\% del P.I.B. Según el I.N.E. (Instituto Nacional de Estadística) España recibió en el año 2012, 57,7 millones de turistas extranjeros. Reino Unido y Alemania son los países que actúan como primeros emisores de turistas con 12,5 millones y 8,8 millones, siendo 
Francia el tercero. Los puntos turísticos con mayor número de pernoctaciones son Madrid, Barcelona y Benidorm. Los países con mayor número de turistas extranjeros recibidos en el año 2012 son Francia con 83 millones, Estados Unidos con $67 \mathrm{mi}-$ llones, y empatado con España, China con 57,7 millones.

La restauración es uno de los servicios principales demandados por todos estos turistas. Un solo dato bastaría para demostrar la relevancia de dicho sector en España. Y es que el restaurante más antiguo del mundo según el Libro Guinness de los Récords está en Madrid (VV.AA. (2014). Guinness World Records. España. Editorial Planeta). Situado en la calle de Cuchilleros, el restaurante Botín lleva abierto al público desde el año 1.725. Personajes como Goya, Hemingway, Pérez Galdós y Truman Capote fueron clientes o citaron en sus obras dicho negocio. El bar más antiguo es el Rinconcillo, en Sevilla, abierto desde 1670.

Según un estudio presentado por Coca Cola en mayo de 2.013 denominado "Vínculo entre los ciudadanos y el bar", en España hay 350.000 establecimientos dedicados a la hostelería. Si hacemos el cálculo con respecto a los 47,2 millones de habitantes del país, nos sale un resultado de un bar por cada 135 personas. Con esta cifra, podemos observar el alto grado de competitividad que existe en el sector. Tras analizar una muestra de más de 2.000 personas, se obtuvieron resultados como el que afirma que un $84 \%$ de los encuestados asocia el bar con las palabras diversión, disfrute o alegría.

Si nos fijamos en los motivos de elección de un bar por parte de los clientes, nos encontramos que con un $22 \%$ prima la calidad de la comida y la bebida. Con un $21 \%$ la amabilidad del personal y con un $14 \%$ el precio de lo ofertado.

Nuevamente, podemos comprobar que el trato del personal y la relación que éste genera con el cliente, es una pieza fundamental para atraer nuevos clientes y fidelizar aquellos que ya tienen.

\section{2- FORMAS DE PROCEDER DE UN RESPONSABLE DE COACHING EN EL MUNDO DE LA HOSTELERÍA.}

Cuando una empresa del sector hostelero contacta con el encargado de llevar a cabo una tarea de coaching, se recomienda que éste siga un proceso ordenado para recaudar información para posteriormente obrar con ella como estime más oportuno. En la primera toma de contacto debemos saber qué empresa es, a qué se dedica, cuántos trabajadores la integran, cuál es su mercado, dónde está situada y qué buscan a nivel general con nuestro trabajo. Los detalles concretos serán requeridos en una segunda entrevista.

Hoy en día, el papel de las redes sociales e internet es fundamental a la hora de recaudar información sobre una entidad dedicada al mundo de la hostelería. En ellas podemos encontrar cientos de comentarios de usuarios que nos aportarán una visión directa de la opinión del consumidor (Mariano Choliz, Clara Marco (2012). Adicción a internet y redes sociales. España. Alianza Editorial). Si se le ha dado un mal servicio a un cliente y éste lo ha difundido por la red, sus palabras pueden condicionar a numerosos posibles futuros clientes. Dar el mejor trato posible será siempre la primera norma. Debemos recordar que clientes descontentos destruyen reputaciones. Por 
tanto, conviene investigar en internet todo lo relacionado con la empresa, para en caso de ser necesario, trabajar en ello posteriormente.

La segunda fase para reclutar información es in situ. Una vez nos encontremos en la zona de influencia de la empresa, deberíamos preguntar a los vecinos, taxistas y transeúntes acerca del local o de los posibles competidores. Por poner un ejemplo, en caso de tratarse de un restaurante, podemos preguntar qué sitios nos recomiendan para ir a comer. Se puede decir que nos han hablado de X (la empresa contratante) y queremos saber qué opinión le merece.

Posteriormente, se aconseja visitar el local como un cliente más. Dado que no nos conocen, podemos anotar valiosa información de las conductas de los trabajadores de primera mano. Tras la acción anterior, mantendremos una reunión con los directivos y con el departamento de recursos humanos para que nos expongan con detalle qué aspectos desean mejorar en su empre$\mathrm{sa}$, dónde creen que tienen sus problemas, cuál es la situación actual y qué objetivos persiguen con nuestra intervención. Como todavía los empleados no nos conocen, podemos volver a visitar el local como un cliente más, pero esta vez observando aquellos detalles que nos han sido requeridos por los directivos de la empresa.

Se deberá estar unos días observando cómo trabajan, cómo se comportan cuando interactúan entre ellos y con sus figuras superiores, pudiendo encontrar posibles conflictos personales que lógicamente habrá que resolver. En la mayoría de ocasiones, el mal entendimiento de un gesto, de la forma de expresar las cosas de uno, es el desencadenante de una mala relación (Musitu, G. (1998). Manual de Psicología de la Comu- nicación. España. Editorial CSV).Con el diálogo y la aclaración, suelen solucionarse la mayoría de los problemas interpersonales que existen en las empresas.

Analizaremos esos datos recogidos y detallaremos cuáles son los puntos fuertes, las carencias, los aspectos a desarrollar a nivel individual y a nivel colectivo y diseñaremos un plan de acción exclusivo, con las actividades, formación y orientación que creamos conveniente. En este punto es fundamental haber definido la personalidad de la empresa, su identidad. Cómo desean ser vistos por el cliente. Existen empresas hoteleras que se caracterizan por el alto poder adquisitivo de sus clientes y les dan así un trato exquisito. La educación y la pulcritud en el trato no tienen por qué estar reñidos con el buen humor y las bromas, pero éstas deberán ser comedidas y aptas a la situación.

A continuación, desarrollaremos las sesiones que estimemos oportunas, tanto con empleados como con los responsables del negocio. La "excusa" de la formación en el desarrollo de habilidades es el mejor marco sobre el que realizar nuestra labor. En ellas podremos observar tanto los roles como las relaciones establecidas. En estas sesiones, evitaremos dejarnos influenciar por el líder (Álvaro, J. L. y Garrido, A. (2003). Psicología social, Perspectivas psicológicas y Sociológicas. España. Editorial MC Graw Hill). Esto significa, que en todo grupo existe una persona con habilidades innatas que le hacen ser el cabecilla del grupo. Algún comentario suyo puede hacer que nuestra opinión con respecto a otros trabajadores quede sesgada. Así mismo, deberemos evitar situarnos siempre en el mismo lugar. Es aconsejable cambiar de posición e incluso mezclarnos cuanto nos 
sea posible en el grupo, para que éste no nos tome ni como un elemento directivo ni con preferencias por estar sentado al lado de alguno de ellos. A la hora de dar charlas formativas, recordaremos por tanto, evitar que nuestra posición y movimientos sean exactamente iguales.

En estas sesiones es donde se persigue desarrollar toda la creatividad posible así como formalizar un clima de trabajo óptimo entre compañeros. Dedicar alguna actividad al Brainstorming o tormenta de ideas, suele ser muy productivo, dado que se definen nuevas líneas de actuación sugeridas por el propio equipo (Eric Maisel (2011). Brainstorming. España. Editorial Obelisco). En la mayoría de las ocasiones, ocurre que tras una primera idea sugerida por alguien, es completada o matizada con algún aspecto que aporta otra persona y que la hace simplemente, genial.

Por último y una vez fijadas las pautas marcadas, nuestra labor será la de supervisar puntualmente que se sigan manteniendo. La función del coaching de equipos es prácticamente un acompañamiento en el aprendizaje de un equipo, puesto que los integrantes del mismo son los que deben dirigir su propio aprendizaje, nuevas tareas que crear y actitudes en el trabajo que mantener para que continúe su correcto desarrollo. Lógicamente nuestras funciones, forma de proceder y forma de cobro, deberán estar claramente descritas y formalizadas legalmente con un contrato.

Independientemente de estos consejos, cada profesional tiene su forma de trabajar y en ocasiones, la innovación y adaptabilidad al terreno son esenciales para desarrollar una labor exitosa.
En resumen, las tareas a llevar a cabo serían:

1- Recogida de datos demográficos.

2- Investigación en internet.

3- Investigación en el contexto y alrededores.

4- Recogida de datos ciega como un cliente más.

5- Reunión con directivos y departamento de RRHH de la empresa contratante y concreción de los aspectos que desean trabajar. Planteamiento de la información recopilada en nuestra recogida de datos ciega.

6- Recogida de datos específicos como un cliente más.

7- Análisis de puntos fuertes y carencias.

8- Determinar la personalidad de la empresa.

9- Plan de acción: Trabajo para el desarrollo individual y desarrollo colectivo.

Acciones.

10- Supervisión y mantenimiento.

\section{3- FORMACIÓN GENERAL PARA EMPLEADOS EN EL SECTOR HOSTELERO.}

La formación continuada en cualquier tipo de empresa y puesto desempeñado, siempre es necesaria. Ésta ha de presentarse tanto para el dueño del negocio como para la totalidad de sus empleados, tanto a nivel individual como colectivo.

Esta necesidad formativa puede ser el escenario perfecto para poder desarrollar el trabajo orientado al coaching a nivel práctico, haciendo uso de las técnicas que se estimen oportunas. Tras haber establecido unos objetivos concretos y haber diseñado 
un plan de acción que permita alcanzarlos, las clases formativas conformarán una serie de encuentros entre los participantes que serán el espacio óptimo a través del cual se lograrán los objetivos finales.

Amparándonos en un marco teórico, podemos dividir el proceso formativo en dos categorías (a nivel individual y a nivel colectivo). En ellas se desarrollarán los procesos de observación de distintos puntos de vista, de toma de conciencia del poder de elección de cada uno, de clarificación de objetivos y de la consiguiente actuación (Cardon A. (2005) Coaching de Equipos. España. Editorial Gestión 2000).

Formación de aspectos para desarrollar a nivel individual:

Toda empresa está formada por un conjunto de individuos. Cada uno de ellos es único, porque única es su vida, sus circunstancias y su estilo de desarrollo dentro de una organización. Debemos por tanto, prestar toda la atención, tiempo y recursos posibles a conocer bien a cada uno de los empleados.

Generar un buen clima de confianza desde la base, es la clave para poder comprender a un trabajador en el amplio abanico de diversas situaciones posibles. Interesarse por su vida personal y estar al corriente en este aspecto, puede ayudarnos a comprender e incluso predecir algunas de sus conductas. Por ejemplo, no podemos esperar el mismo rendimiento de una persona a la que todo le va muy bien en su relación de pareja, que de una persona en plena ruptura matrimonial.

No todo el mundo nace aprendido y menos con el don de saber desarrollar una profesión a la perfección. Por consiguiente, evaluaremos en qué aspectos o conocimientos el trabajador puede necesitar mayor refuer- zo para la correcta realización de las tareas del puesto.

Muchos altos directivos creen que los que necesitan formación son sus empleados, sin ni siquiera contemplar la posibilidad de que también ellos deberían de mejorar en algunos aspectos.

Para ser el responsable de un negocio en la hostelería, al igual que en otras muchas ramas, es altamente recomendable conocer todos los aspectos relacionados con cada puesto de trabajo (Chowdhury, S. (2006). El Fabricante de Helados. España. Editorial Empresa Activa). Un gran número de empresas, a la hora de formar a un trabajador que va a dirigir a una gran parte de sus empleados, les hacen estar un tiempo desempeñando sus funciones. Empezar desde abajo. Esta es una forma de lograr que cuando se tenga el mando directivo, se conozca realmente qué se le puede exigir a un trabajador, las ventajas y desventajas del puesto, y cómo se sienten los que ocupan esa posición, logrando así una mayor empatía. El problema radica en que tras unos años siendo directivo, se olvida con facilidad el pasado y las funciones de algunos puestos inferiores (Costa, M. y López, E. (2002). Los secretos de la dirección. España. Editorial Pirámide). Es por esto que es altamente recomendable que reciban parte de la formación y sean partícipes de algunas de las sesiones de coaching.

Además, hay que remarcar que el jefe del establecimiento debe dar siempre ejemplo a sus trabajadores. Para ellos es su figura de referencia. Un jefe que siempre está nervioso, estresado por querer dar un buen servicio y que apenas sonríe a los clientes es lo peor que le puede pasar a un local, dado que solemos adoptar e imitar comportamientos de nuestros semejantes. Se puede 
estar igual de atento y actuar de forma disciplinada con una sonrisa en el rostro.

Formación de aspectos para desarrollar a nivel colectivo (el equipo).

Cuando una persona pertenece a un grupo, tiende a pensar y actuar de forma parecida a los demás. Este contagio, viene determinado por la sugestión de la que hablaba Sigmund Freud, por el hecho de querer pertenecer al grupo, de ser igual que los demás. (Sigmund Freud (2010). Psicología de las masas. España. Alianza Editorial). De esta forma, sabemos que generando un espíritu de grupo alegre y contento, tendremos a unos individuos que disfrutan de sus horas de estancia con este grupo. Esta situación o fenómeno es precisamente lo que se desea generar. La clave del éxito radica en que el cliente capte y sea a la vez partícipe de este mismo sentimiento de felicidad.

La mayoría de clientes del sector del turismo, tienen como objetivos principales, pasarlo bien y descansar. Demandarán por tanto un clima agradable, en el que todo va bien y en el que la alegría reine. La percepción de estrés por parte de los trabajadores o conflictos internos entre ellos, puede arruinar este clima en décimas de segundo, con lo que los enfrentamientos y desavenencias entre compañeros estarán "prohibidas". La búsqueda y existencia de un chivo expiatorio en los grupos grandes suele ser normal (Pinillos,J.L.(1981). Tres psicólogos en el bosque animado. España. Editorial Alfaplus). Poder echar las culpas a alguien y evitar así las responsabilidades, es algo innato a las formaciones numerosas, con lo que de igual forma, habrá que prestar atención a la posible existencia de tal figura y una vez hallada, trabajar para que deje de serlo. No sólo se persigue alcanzar un buen clima, se busca lograr ser un equipo. Y en un buen equipo, todo el mundo es responsable, porque todos pertenecen a una misma entidad y así deben de sentirlo.

Los aspectos que se trabajarán en la formación estarán relacionados sobre todo con la comunicación, tanto verbal como no verbal. En relación a la primera, se hará énfasis en cómo desarrollar una correcta escucha activa, mientras que en la segunda, se incidirá en el reconocimiento facial y de las diferentes expresiones, gestos y disposición en el espacio con respecto al cliente y viceversa.

\section{4- MOTIVACIÓN DEL EQUIPO.}

Cada día, millones de personas se levantan desmotivadas para ir a sus trabajos. Sin duda alguna, la desmotivación es el comienzo de una de las enfermedades que más bajas laborales causa en este siglo, la depresión.

Debido a la actual situación económica en la que tener un trabajo es todo un lujo, la mala praxis en el mismo es algo a lo que se le resta importancia de manera racional.

Estamos en una nueva etapa en donde la gente ocupa puestos que no le corresponden bien sea por enchufismo o por necesidad, en donde no se percibe la cantidad de dinero acorde a un plan de vida normal y en donde aceptar y resignarse es la única solución. La incoherencia mental que genera estar agradecido y sentirse desmotivado al mismo tiempo, puede generar un estado de incomodidad permanente en el empleado.

La clave para estar a gusto en un trabajo se encuentra en el contexto inmediato y diario. El buen clima con los compañeros es la base del éxito empresarial. Tener a todo el equipo de trabajadores contento será por 
tanto uno de los principales objetivos que perseguir como responsable de la tarea del coaching.

Cuando le preguntas a las personas sobre lo más importante en su vida, el 99\% contestará que su familia. Al final, lo más relevante y lo que de verdad nos llena es la vida personal (Eduardo Punset (2007). El viaje a la felicidad. España. Editorial Destino). Pero en el trabajo solemos estar una media de entre 8 y 11 horas al día durante 5 o 6 días de la semana. Ya que se pasa una gran parte de la vida en dicho lugar, será lógico hacerlo o al menos intentarlo hacer de una forma lo más agradable y gratificante posible. Es ahí donde debemos escoger el tener la mejor actitud.

En palabras de Confucio, "Elige un trabajo que te guste y no tendrás que trabajar ni un día en toda tu vida”. Sería genial que todo el mundo pudiera acceder y desempeñar el rol laboral con el que soñó desde pequeño, pero por desgracia, la realidad de las diversas circunstancias de la vida, conducen a que un gran número de personas no lo logren. Sin embargo, existen pequeños trucos sin coste económico alguno, para convertir un aburrido día de trabajo en una jornada agradable, motivadora, llena de nuevos retos y entretenimiento.

\section{Inicio del buen clima laboral:}

Entrar con una sonrisa al trabajo es el primer paso para generar un bucle de continuo bienestar. La sensación de un ambiente agradable es una mera percepción que está condicionada a la conducta de los compañeros y a como uno mismo la interprete.

Instaurar el tener una actitud positiva y optimista en lugar de rumiar pensamientos de corte deprimente, debería ser el primer objetivo del día. Se debe procurar transmitir el concepto que sea cual sea la labor que se ha de desempeñar, ésta será experimentada como uno desee. ¿Por qué no probar a ver "la vie en rose"? Pensar y visualizar la propia actitud que muestra la mayoría de trabajadores los viernes, suele ayudar. Hay que recordar que el cómo se afronta un hecho o se inicia un día es una elección de una actitud. Podemos escoger una actitud negativa ante la vida o podemos elegir que sea positiva. Es obvio que ésta segunda aportará muchos más beneficios personales que la primera.

Algunos profesionales hacen uso de visualizaciones de videos o proyecciones en las que se muestran escenas de humor, o incluso escenas en las que aparece gente con una risa contagiosa. El acto reflejo de sonreír o incluso reír, es quien se encarga del resto. Para este tipo de acciones, suele venir bien consultar algunos manuales de risoterapia.

\section{Trabajo y juego:}

Lograr un entorno favorable suele ser fácil siempre y cuando los trabajadores perciban que son bien tratados por todos los compañeros y por los superiores. La ausencia de mobbing y la existencia de unas pautas bien definidas de respeto mutuo son esenciales. Una vez logrado esto, se puede empezar a instaurar ciertas reglas que conviertan los quehaceres en un juego (Stephen C. Lundin, M.D. Harry Paul y John Christensen con Philip Strand (2002).Historias de FISH. España. Editorial Empresa Activa). Concursos en los que se premie con un día libre a los mejores vendedores, papeleras con cestas de baloncesto en los días de limpieza de escritorio o celebrar con una carrera de sillas un nuevo contrato con una gran empresa, pueden ser acciones que aporten mucho mayor entretenimiento a los días. 
Cuanto mayor sea el repertorio de actividades lúdicas, menos aburrida será la jornada. Fuera de la misma, se pueden crear equipos o algún tipo de competición, pero procurando evitar que sean deportes de contacto. Cuando se practican este tipo de deportes a una edad avanzada suele ocurrir que físicamente no se está igual de bien que años atrás, con lo que un factor que aparece es la frustración en el terreno físico. Este hecho, acentúa que posibles choques se agraven no sólo por la consecuencia física del golpe, sino por la mala intención de algún jugador, que aprovecha la oportunidad para vengarse de un compañero por alguna mala "jugada" del pasado en lo laboral.

\section{Agradecimiento:}

A todo el mundo le gusta saber que su trabajo está bien hecho. El reconocimiento por parte de un superior o incluso de un compañero en el momento preciso, suele funcionar muy bien a nivel emocional y sobre todo como un refuerzo de la conducta premiada.

Alegrar el día de otra persona no sólo le hace sentir bien al causante de la acción sino que al modificar el estado anímico del otro, se influirá indudablemente en el ambiente que los rodee. Encontrar un hueco al final de la quincena o incluso del mes, en donde una vez reunidos todos, se resalten los aspectos positivos mostrados o logros conseguidos, es un refuerzo para todos los partícipes del mismo. Además de generar bienestar, se creará así mayor sentimiento de equipo.

\section{Ser visualmente un equipo:}

Todos los equipos deportivos se caracterizan por una cosa. Todos sus integrantes portan el mismo uniforme que además de darles una entidad, los diferencia del resto. Existen muchos establecimientos en donde la vestimenta se deja a elección del propio trabajador, y aunque pueda parecer que gozan de mayor libertad, a la larga se perjudica el sentimiento de pertenencia a un equipo. Además, hay que recordar que también conviene que cada uno sepa cuál es su puesto. El cocinero, el camarero y el dueño deberían diferenciarse por su ropa de trabajo. Ponerse de acuerdo en portar una vestimenta de color neutro como el negro, no resulta complicado.

\section{Correcta comunicación:}

Sentirse escuchado es uno de los hechos más demandados por los trabajadores. La falta de tiempo, las prisas, las puertas cerradas o secretarias que intermedian para dar cita con un superior son obstáculos comunes que dificultan el correcto flujo de comunicación.

Algunas empresas tienen una jornada de puertas abiertas en donde los empleados pueden presentar sugerencias, quejas y nuevas ideas de proyectos a los directivos, encontrándose así mucho más motivados gracias al sentimiento de aportar algo más que trabajo a la empresa. Es aconsejable que exista la percepción de la presencia de una comunicación bidireccional, de feedback y de recibir información general sobre los objetivos, resultados y política o estrategias de futuro de la compañía. Esto eliminará de raíz los posibles rumores que, en ocasiones, se crean fruto de la desinformación de los

trabajadores sobre un aspecto que les afecta directamente. Habrá que asegurarse por tanto, de la existencia de una comunicación correcta. 


\section{Importancia del trabajo:}

Además de recibir reconocimiento por lo bien hecho, el ser humano necesita saber que existe un peldaño más al que puede acceder, porque es en el progreso mismo, donde radica nuestra evolución como especie (J.B. Bury. (2009). La idea del progreso. España. Alianza Editorial). Las posibilidades de promoción ofrecen además de tener una meta, obtener una gran satisfacción al comprobar que se avanza en la correcta dirección. Delegar responsabilidades en los empleados, suele facilitar este proceso al dotar de mayor autonomía a los empleados, además de incentivarlos a usar su creatividad, sintiéndose de esta forma, absolutos responsables de sus decisiones. La mayoría de los responsables o altos directivos son reacios a delegar tareas. Si logramos hacerles ver que además de las ventajas anteriormente descritas, dispondrán de mayor cantidad de tiempo para realizar otras actividades, podremos obtener el resultado deseado con mayor probabilidad.

\section{Ser la empresa:}

Muchas de las grandes empresas de reconocido nombre, logran que sus trabajadores se involucren con la firma que representan a través de las ventajas personales que eso supone. El sentimiento de marca puede lograrse a través de la celebración de los éxitos de la compañía, con importantes descuentos con empresas filiales o incluso regalando a los trabajadores diversos productos manufacturados en la propia empresa. Hacerlos partícipes de los beneficios de forma inmediata les proporcionará cierta empatía con los puestos directivos, además de obviarles la necesidad de una orientación clara hacia objetivos bien definidos. Por otra parte, el hecho de hacerles consu- midores habituales del producto, no sólo aumentará el sentimiento de familiaridad con la marca, sino que podrán aportar nuevas ideas de mejora desde el punto de vista del consumidor.

\section{5- LAS REDES SOCIALES, INTERNET Y EL COACHING 2.0.}

Hoy en día, vivir sin internet o estar sin cobertura es casi una utopía. Cada año, aparecen nuevas redes sociales o formas de comunicación entre sus usuarios y consumidores. La aparición de estas nuevas aplicaciones se debe al mayor aumento del número de consumidores, dado que éstos se inician en el mundo digital a edades más tempranas. De hecho, este fenómeno ha dado lugar a la aparición de una nueva subdivisión social, estando los denominados inmigrantes digitales, nacidos entre 1.940 y 1.980 y los nativos digitales que son nacidos entre 1.980 y 1.990 (Marc Prensky (2011). Enseñar a nativos digitales. España. Ediciones SM). Personalmente, me atrevo a sugerir el nombre de endo-digitales para la nueva generación que nacidos a partir de 1.990-1.995, ya poseen "unas habilidades prácticamente innatas muy superiores al resto en el manejo de lo tecnológico" (tablets, smart phones, smart tvs, etc).

Según estudios realizados por una compañía llamada Experian Marketing Services, los norteamericanos pasan en las redes sociales un promedio de 16 minutos de cada hora, lo que se traduce en una cuarta parte del día (unas 6,4 horas al día).

El triunfo de plataformas como Facebook, Twitter, Instagram, Wechat y otras radica en las necesidades del ser humano. Por una parte y como seres sociables que somos, la comunicación con nuestros semejantes. En segundo lugar y como seres egocéntricos 
que somos, la necesidad de aprobación y reconocimiento, el orgullo.

Pero obviamente, también ha supuesto un avance cuantitativo y cualitativo en nuestra manera de establecer y mantener relaciones al igual que al abrir nuevas opciones en el terreno profesional. De ahí que me atreva a denominar la figura del encargado del coaching 2.0 como aquel que se sirve de las nuevas herramientas tecnológicas para desarrollar su labor.

No cabe duda de la necesidad presencial in situ para realizar la actividad de coaching con una empresa (bien sea del sector hostelero $\mathrm{u}$ otros). Pero el seguimiento y el mantenimiento de nuestra labor, también se puede desarrollar en la distancia gracias a las video-llamadas o incluso a la mensajería instantánea. En el coaching 2.0 se tiene la posibilidad o se puede seguir orientando a un equipo de dos formas. La primera, a nivel individual, de forma privada e interactuando de forma exclusiva con el grupo. La segunda, creando un perfil común a todos los clientes / empresas / coachees en donde se irá subiendo material que pueda ser utilizado o aprovechado por quien lo desee. La apuesta más segura en la actualidad para este tipo de acciones es el formato del vídeo corto en duración. En este caso, aportamos algo que nos diferencia del resto, la constante innovación y el sustento de lo trabajado en el pasado. Con el paso del tiempo, es normal que se tienda a olvidar lecciones aprendidas del pasado.

Y todavía puede ser más difícil cuando hablamos de emociones, ya que éstas fluctúan sin cesar. Una sola palabra o una sola imagen pueden activar el inicio de aquella actitud deseada o aprendida en el pasado.

Al ser un canal bidireccional las redes sociales también pueden ser un buen escapa- rate en donde veamos reflejada (a través de sus fotos, vídeos, reuniones, consecución de objetivos empresariales, etc.) nuestra labor hecha con el grupo y cómo ésta avanza, pudiéndola corregir con un comentario o valorarla positivamente con un simple me gusta.

\section{3.- CONCLUSIONES.}

El éxito en cualquier negocio hostelero radica en dar un buen servicio. Para ello, el personal debe poseer ciertas habilidades profesionales así como disponer de diversos recursos personales que mostrar en la interacción con el cliente. Pero ni una ni otra cosa se puede lograr sin la base adecuada, sin unos cimientos fuertes y sin un estado psicológico que predisponga a tener una actitud positiva.

El actor principal de coaching en el sector de la hostelería y el turismo será el responsable de lograr su optimización a nivel personal para que éstos puedan llegar a la plena satisfacción tanto en su vida privada como en la laboral.

Ejercerá como guía desde el inicio del contacto con todos los participantes del proceso. Analizará y detectará los puntos débiles y fuertes individuales así como manejará los diferentes estilos de relaciones personales entre ellos. Dotará a los asistentes de los recursos que estime oportuno y perseguirá como último fin la consolidación de un equipo de trabajadores motivados en su tarea.

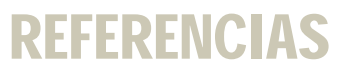

Álvaro, J. L. y Garrido, A. (2003). Psicología social, Perspectivas psicológicas y Sociológicas. España. Editorial MC Graw Hill. 
Musitu, G. (1998). Manual de Psicología de la Comunicación. España. Editorial CSV.

Stephen C. Lundin, M.D. Harry Paul y John Christensen con Philip Strand (2002).

Historias de FISH. España. Editorial Empresa Activa.

Cardon A. (2005) Coaching de Equipos. España. Editorial Gestión 2000.

Costa, M. y López, E.(2002). Los secretos de la dirección. España. Editorial Pirámide.

Chowdhury, S. (2006). El Fabricante de Helados. España. Editorial Empresa Activa.

Gistain Montolio, C. (2012). Coaching en el sector del turismo y la hostelería.

Apuntes del Máster en Coaching y Liderazgo de la Universidad de Valencia ( $\sin$

publicar).

Sigmund Freud (2010). Psicología de las masas. España. Alianza Editorial.

Pinillos,J.L.(1981). Tres psicólogos en el bosque animado. España. Editorial Alfaplus.

Eduardo Punset (2007). El viaje a la felicidad. España. Editorial Destino.

Marc Prensky (2011). Enseñar a nativos digitales. España. Ediciones SM.

VV.AA. (2014). Guinness World Records. España. Editorial Planeta.

Eric Maisel (2011). Brainstorming. España. Editorial Obelisco.

J.B. Bury. (2009). La idea del progreso. España. Alianza Editorial.

Mariano Choliz, Clara Marco (2012). Adicción a internet y redes sociales. España. Alianza Editorial.

Fecha de recepción: 9/12/2013

Fecha de aceptación: 2/04/2014 